\title{
The effect of polyethylene glycol adhesion barrier (Spray Gel) on preventing peritoneal adhesions
}

\author{
Dasiran $\mathrm{F}^{1}$, Eryilmaz $\mathrm{R}^{2}$, Isik $\mathrm{A}^{3}$, Okan $\mathrm{I}^{1}$, Somay $\mathrm{A}^{4}$, Sahin $\mathrm{M}^{1}$ \\ Department of General Surgery, Erzincan University, Erzincan, Turkey. kararda@yahoo.com
}

\begin{abstract}
The prominent cells in the late phase of wound healing during proliferation and matrix deposition are fibroblasts. Foreign materials in the operation site like prosthesis prolong the inflammation and induce fibroblast proliferation (8). 3 different prostheses used in this study induced chronic inflammation and fibrosis and provided an effective repair. Dense and thick adhesions due to fibrosis also induced strong adhesions to omentum and small intestine if only polypropylene mesh used for hernia repair. However, there was no difference between SprayGel treated polypropylene mesh and Sepramesh when compared for fibrosis. It also prevents the intraabdominal adhesion formation. It is nontoxic, sticky adherent, non- immigrant and easy to use both in open and laparoscopic surgeries. This experimental study revealed that polyethyleneglycol applied polypropylene mesh accomplishes hernia repair with significantly less adhesion formation than polypropylene mesh alone while securing a remarkable economy than adhesion barrier coated dual meshes (Tab. 6, Fig. 7, Ref. 23). Text in PDF www.elis.sk.

Key words: abdominal surgery, adhesion, postoperative morbidity, hernia, barrier, wound healing, fibrosis.
\end{abstract}

\section{Introduction}

Postoperative adhesions still remain a challenging problem for modern medicine concerning all branches of surgery. Adhesion formation occurs after almost all abdominal operations regardless of the technique or method used. Intestinal obstructions, chronic abdominal pain, difficulty with relaparotomy procedures and infertility in women are major postsurgical morbidities associated with adhesions (1)

Ventral hernias maybe congenital, acquired, incisional or traumatic in origin and the treatment is mainly surgical. The repair can be done primarily or by using prosthetic materials. Failure rates up to $58 \%$ are reported with primary repair in literature (1-4). The main reason for the increased failure rate is due to the dehiscence of fascia that cannot hold the stitches in place against abdominal pressure. Prosthetic materials are used for a tension-free repair. The most commonly used prosthetic material is polypropylene mesh, which causes dense adhesions if placed in the abdomen. Hernia repair with prosthesis reports less complication and recurrence rates (5).

Research for the prevention of adhesion mainly focuses on surgical techniques, pharmacologic agents and physical barriers. Surgical techniques for the prevention of adhesions aim at minimal surgical trauma, smaller incisions, more laparoscopic practices, adequate haemostasis, prevention of infection and less foreign material in the

'Department of General Surgey, Gaziosmanpasa University, Tokat, Turkey, ${ }^{2}$ Department of General Surgery, Akdeniz University, Antalya, Turkey, ${ }^{3}$ Department of General Surgery, Erzincan, Turkey, and ${ }^{4}$ Department of Pathology, Fatih Sultan Mehmet Training and Research Hospital, Istanbul, Turkey

Address for correspondence: A. Isik, MD, Department of General Surgery, Erzincan University, Erzincan, Turkey.

Phone: +905330580707 operation site (suture materials, prosthesis...) (6). Pharmacological agents including corticosteroids, antihistamines, non-steroidal antiinflammatory drugs, fibrinolytics, vitamin E, calcium antagonists, interferon, anticoagulants, progesteron-estrogen, taurolin, halofujinon, L- arginine, pentoxifilline, interleukins and aprotinin were tested for different phases of inflammation and adhesion formation (7-9). Although many agents have been promising to reduce the rates of adhesion formation, clinical application is still lacking. Adhesion barriers physically separate traumatised tissues during the healing period. An ideal adhesion barrier should be chemically inert, nontoxic, non carcinogenic, non allergic, easy to use, applicable for laparoscopic procedures, resistant to mechanical stress, cost-effective and available in different forms and dimensions (10). Physical barriers may be liquid or gel-form. Crystalloid solutions, dextran 70, hyaluronic acid, hyaluronic acid-phosphate tamponade, carboxymethylcellulose and polylactic acid were reported to reduce the adhesion formation (11-14). Hyaluronate carboxymethylcellulose based bioresorbable membrane is successfully used to prevent postoperative adhesion. Although it has been shown to be effectivein many studies, the high cost hinders its wide use (15).

Synthetic gel barriers gather the liquid molecules with hydro flotation effect and prevent the direct contact of the serosa and peritoneum. Mechanical sheathing like a cover also separates the traumatised tissues (16). The adhesion barriers remain intact for one to four weeks and then hydrolysed, absorbed and eliminated from the body. Spray Gel (Confluent SurgicalInc., Waltham, MA) is a synthetic, modified polyethyleneglycol-based absorbable adhesion barrier. It consists of two different precursor liquids that solidify when mixed. One precursor contains a dilute concentration of methylene blue simplifying the visualisation of hydrogel and estimation of thickness. Polyethyleneglycol adhesion bar- 
rier remains strongly adhered to the site of application for 5 to 7 days, after which it hydrolysed and cleared from the kidneys. This sprayable hydrogel can be easily applied at laparoscopy with special reusable pump sprayer designed for laparoscopic operations.

In the present study, we evaluated the effectiveness of polyethylene glycoladhesion barrier (SprayGel) coated polypropylene mesh by comparing hyaluronate carboxymethylcellulose based bio resorbable membrane (Sepramesh) to prevent postoperative adhesion in an experimental incisional hernia model.

\section{Materials and method}

This experimental study is done in Istanbul University Experimental Research Laboratory with the consent of Experimental Animals Ethical Committee. Twenty seven Wistar Albino rats weighting 200-250 g were used for the study. Rats were fed in animal cages and ad libitum.

\section{Surgical technique}

The rats were anesthetized with $50 \mathrm{mg} / \mathrm{kg}$ intramuscular ketamine hydrochloride (Ketalar) injection under sterile condition. Hairs in midline were carefully shaved and povidone iodine (Isosol) was applied for antisepsis. $100 \mathrm{mg} / \mathrm{kg}$ ampicilline-sulbactam was intramuscularly injected for antibiotic prophylaxis. After a $4 \mathrm{~cm}$-length midline incision, the skin and subcutaneous tissue were dissected from underlying fascia. A fascia defect of $3 \times 2 \mathrm{~cm}$ in size was created together with the excision of fascia and peritoneum.

\section{Tab. 1. Adhesion grading (Jenkins et al).}

\begin{tabular}{cl}
\hline Grade & Adhesion formation \\
\hline 0 & No adhesion \\
1 & Loose adhesion needs blunt dissection \\
2 & Adhesion needs aggressive blunt dissection \\
3 & Dense and thick adhesion needs sharp dissection \\
\hline
\end{tabular}

\section{Tab. 2. Fibrosis grading (Hooker et al).}

\begin{tabular}{cl}
\hline Grade & Fibrosis score \\
\hline 0 & No fibrosis \\
1 & Minimal \\
2 & Moderate \\
3 & Dense \\
\hline
\end{tabular}

Tab. 3. Inflammation grading (Hooker et al).

\begin{tabular}{cl}
\hline Grade & Inflammation score \\
\hline 0 & No inflammation \\
\hline 1 & Giant cells, scattered few lymphocyte and plasma cells \\
\hline 2 & $\begin{array}{l}\text { Giant cells, increased amount of lymphocyte, neutrophil, eo- } \\
\text { sinophil and plasma cell }\end{array}$ \\
\hline 3 & $\begin{array}{l}\text { A great number of mixed inflammatory cells, } \\
\text { Micro abscess formation }\end{array}$ \\
\hline
\end{tabular}

\section{Tab. 4. Macroscopic evaluation.}

\begin{tabular}{ccccc}
\hline Adhesion score & Group 1 & Group 2 & Group 3 & Total \\
\hline 0 & - & - & - & - \\
1 & $3(11 \%)$ & $7(25 \%)$ & $8(30 \%)$ & $18(66 \%)$ \\
2 & $4(15 \%)$ & $2(7 \%)$ & $1(4 \%)$ & $7(26 \%)$ \\
3 & $2(7 \%)$ & - & - & $2(7 \%)$ \\
\hline Total & 9 & 9 & 9 & 27 \\
\hline
\end{tabular}

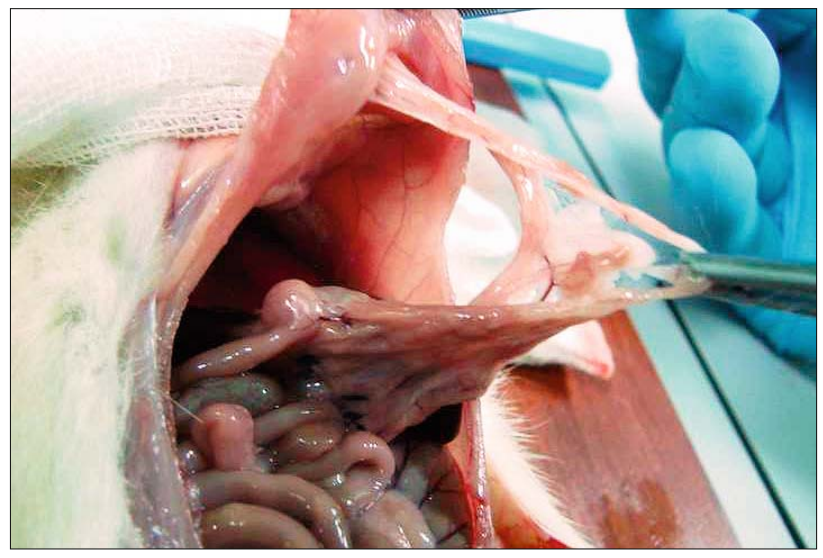

Fig. 1. Grade I adhesion.

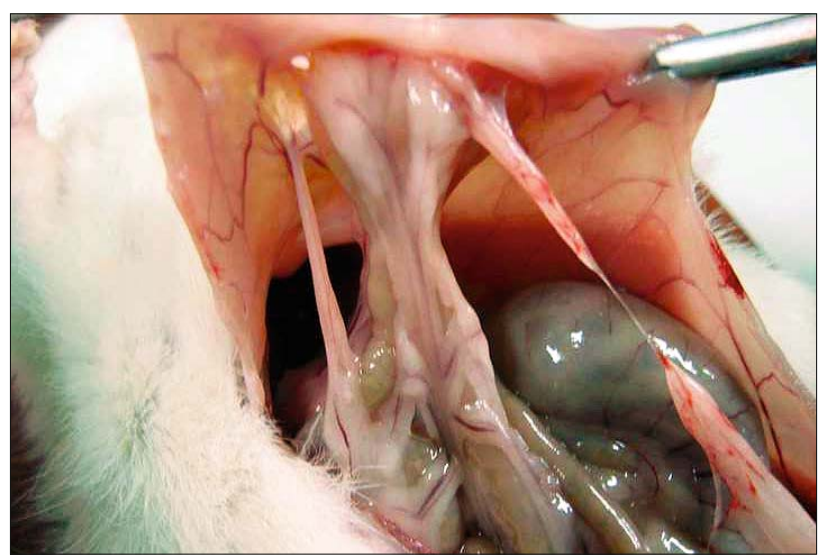

Fig. 2. Grade II adhesion.



Fig. 3. Grade III adhesion.

The antimesenteric surfaces of the caecum and terminal ileum were scraped with dry gauze until serosal petechial bleedings occured. Abdominal wall defects were immediately repaired. Rats weredivided into thre egroups according to the material used for repair:

Group 1: Repair with polypropylene mesh (Prolene, EthiconInc., Somerville, NJ)

Group 2: Repair with polypropylenemesh treated with 0.3 ccPolyethylene glycol adhesion barrier (Spray Gel, Confluent SurgicalInc., Waltham, MA) 
Group 3: Repair with hyaluronate carboxymethylcellulosebased bio resorbable membrane(Sepramesh, Genzyme Surgical Prod., Cambridge, MA)

The same size prosthesis of $4 \times 3 \mathrm{~cm}$ was fixed to fascia with separated $4-0$ polypropylene sutures in all animals. The abdominal incisions were closed with 4-0 silk sutures in a continuous manner. All rats were sacrificed on 28th postoperative day with intra cardiac injection of high dose ketamine hydrochloride. ReverseU incision was performed to view the adhesions. Abdominal front wall flaps were drawn towards caudally and the adhesions between abdominal wall and organs were graded through the scale defined by Jenkins et al (17) (Tab. 1).

\section{Histopathological examination}

En block excised prosthesis and abdominal wall were fixed in $10 \%$ formaldehyde, immersed in paraffin after dehydration. Sections of $5 \mathrm{~mm}$ thickness from the paraffin molds were prepared, stained with Hemotoxylene-Eosin (H\&E) and examined with light microscope to evaluate the fibrosis and inflammation in accordance with grading defined by Hooker et al (Tabs 2 and 3) (18).

\section{Statistics}

Statistical evaluation was performed with the aid of SPSS (Statistical Package for Social Sciences) for Windows 15.0 program. Data was assessed by the means of chi-square test. Results were assessed at a 95\% confidence limit and the "p"value was interpreted as significant when less than 0.05 and highly significant when less than 0.01 . The correlation within the groups were studied with spearman correlation analysis.

\section{Results}

Macroscopically, all rats exhibited evaluation revealed adhesions between abdominal wall and intra-abdominal organs to varying degrees (Figs 1-3). Adhesion score of group1 was significantly higher than group $2(\mathrm{p}=0.04)$ and group $3(\mathrm{p}=0.01)$, whereas Group 2 and 3 adhesion scores were similar $(p=0.6)$. The details of adhesion scores were shown in the Table 4.

Histopathological examinations of abdominal wall and prosthesis revealed the inflammatory cell infiltration, fibrinous exudates, capillary proliferation and micro abscesses in all specimens (Figs 4 and 5). No significant difference was noted among the groups with regard to inflammation. The numerical details of inflammation score was depicted in Table 5.

Masson Trichrome staining was performed on the H\&E stained pieces to evaluate the fibrosis (Figs 6 and 7). Table 6 shows the fibrosis scores of the groups. There was significantly more fibrosis formation in the group 1 . When binary compared group 1 vs. group 2 and group 1 vs. group 3, the differences were significant $(\mathrm{p}=0.003, \mathrm{p}=0.001$, respectively). Group 2 and 3 had a similar fibrosis level $(\mathrm{p}=0.6)$.

There were significant differences among the groups with regard to the costs of the prosthetic materials. Prolene mesh prepared $4 \times 3 \mathrm{~cm}$ cost $2 €$ in the group 1, the same size prolene mesh +0.3 cc SprayGel cost $42 €$ in the group 2, where the same size Sepramesh cost $114 €$ in the group 3 .

\section{Discussion}

This experimental study showed that polyethyleneglycol-based absorbable adhesion barrier (SprayGel) treated polypropylene was comparable to hyaluronate carboxymethylcellulose based bio resorbable membrane (Seprafilm) regarding the adhesiveness to intra-abdominal organs and reduced cost-effectiveness. Spray Gel treated polypropylene mesh induced less adhesions between intra-abdominal organs and mesh in accordance with less induction of inflammation and fibrosis with less cost.

Giant abdominal wall defects resulting after tumor and trauma operations or infections can't be closed primarily. Open abdomen procedures or prosthetic materials are used for large abdominal wall defects. Polypropylene mesh is the most commonly used prosthesis so far. It has the maximum tension force when compared to other prostheses due to intense inflammatory reaction promoted within tissues. However, intra-abdominal organs adhere to the mesh even being separated with peritoneum exactly for the same reason. Therefore, morbidities like chronic pain, intestinal obstruction, fistulas and infertility may necessitate rehospitalisation and even relaparotomies in some cases (19). These morbidities burden additional charge on the national economy. Any method or instrument, which prevents the adhesion formation will not only save many patients from severe morbidities and mortalities but also decrease the economic burden caused by adhesion (20).

Abdominal surgery is extremely common in western world. In 2645 autopsies, Beartet al. inLos Angeles found the incisional scars of previous abdominal operations in $32 \%$, this figure rose to $44 \%$ in

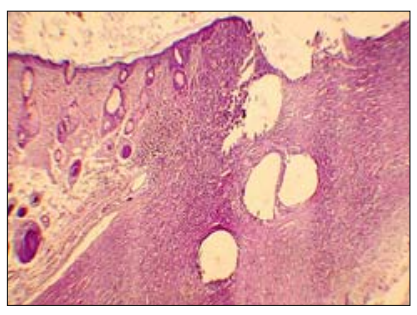

Fig. 4. Foreign body reaction on prosthesis, chronic inflammatorycells, HE x40.

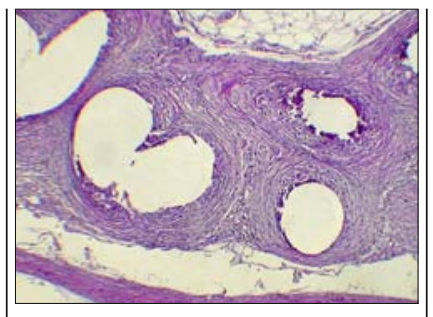

Fig. 5. Chronic inflammation and fibrosis in subcutaneous adipose tissue, HEx100.
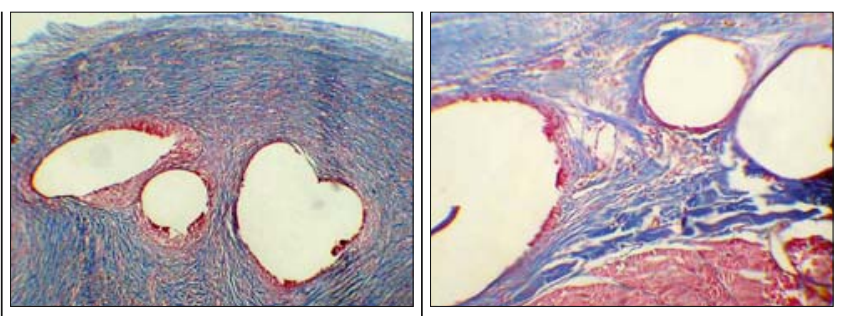

Fig. 6. Masson Trichrome staining

for fibrosis MT x100.

Fig. 7. Fibrosis formation between striated muscle and mesh, MT x400. 
those over sixty years of age. The incidence of abdominal surgery was higher in women than in men (21). We might estimate that approximately one third of adult population has intra-abdominal adhesions albeit majority of them are without symptoms.

Colorectal surgery, appendectomy and gynaecologic operations are the commonest operations that lead to adhesions. Miller and colleagues in the study with 410 acute intestinal obstruction operations due to adhesions reported that former operation was colorectal surgery in $24 \%$, gynaecologic operation in $22 \%$, hernioraphy in $15 \%$ and appendectomy in $14 \%$ (22). The similar study by Williams et al with 201 patients reported the former operation was colorectal surgery in $34 \%$, gynaecologic operation in $28 \%$, appendectomy in $14 \%$, cholecystectomy in $12 \%$,hernioraphy in $8 \%$ and gastric operations in $5 \%$ (23).

Chronic inflammation and fibrosis are intentionally expected when prosthetic material is used for hernia repair. However, sometimes the inflammation extends beyond the anterior abdominal wall through the abdominal organs causing undesirable side effects such as fistula. Prevention of direct contact of the mesh with intra-abdominal organs is critical especially at the inflammatory phase of wound healing (8). The goal to use the Spray Gel treated polypropylene mesh in this experimental adhesion model is to achieve an economic and effective way for prevention of adhesions.

The prominent cells in the late phase of wound healing during proliferation and matrix deposition are fibroblasts. Foreign materials in the operation site like prosthesis prolong the inflammation and induce fibroblast proliferation (8). 3 different prostheses used in this study induced chronic inflammation and fibrosis and provided an effective repair. Dense and thick adhesions due to fibrosis also induced strong adhesions to omentum and small intestine if only polypropylene mesh was used for hernia repair. However, there was no difference between Spray Gel treated polypropylene mesh and Sepra mesh when compared for fibrosis. It also prevented the intra-abdominal adhesion formation. It is nontoxic, sticky adherent, non- immigrant and easy to use both in open and laparoscopic surgeries. This experimental study revealed that polyethyleneglycol applied polypropylene mesh accomplished hernia repair with significantly less adhesion formation than polypropylene mesh alone while securing a remarkable economy than adhesion barrier coated dual meshes.

\section{References}

1. Hodgson NC, Malthaner RA, Ostbye T. The search for an ideal method of abdominal fascia closure; a meta-analysis. Ann Surg 2002; 231: 436-442.

2. Burger JWA, Halm A, Wijsmuller AR, Raa S, Jeekel J. Evaluation of new prosthetic meshes for ventral hernia repair. Surg Endosc 2006; 20: 1320-1325.

3. Caldironi MV, Romano M, Bozza F et al. Progressive pneumoperitoneum in the management of giant incisional hernias: A study of 41 patients. Br J Surg 1990; 77: 306-308.

4. Paul A, Karenkov M, Peters S et al. Unacceptable results of the mayo procedure for repair abdominal incisional hernias. Eur J Surg 1998; 164 : 361-367.

5. Sikkink CJJM, Vriesde Reilingh TS, MalyarAW et al. Adhesion formation and reherniation differ between meshes used for abdominal wall reconstruction. Hernia 2006; 10: 218-222.
6. Ellis $\mathbf{H}$. The cause and prevention of postoperative intraperitoneal adhesions. Br J Surg 1982; 69: 241-243.

7. Censur Z. Postoperatif intraperitonealy apisşikliklarinönlenmesindedeg isikdozlardaki heparin ve Seprafilminetkinliklerininkarsilastirilmasi (tez). Istanbul: Dr. Lütfi Kirdar Egitimve Arastirma Hastanesi2005.

8. HoltzG. Prevention and management of peritoneal adhesions. Fertil Steril 1984; 41 (4): 497-504.

9. Arikan S, Adas G, Barut G et al. An evaluation of low molecular weight heparin and hyperbaric oxygen treatment in the prevention of intra-abdominal adhesions and wound healing. Am j Surg 2005; 189: 155-160.

10. Read RC, Grose WE. Ventral and incisional Hernias. Ed: Nyhus LM. In Schackelford's Surgery of the Alimentary Tract. WB Saunders Co, Philadelphia, 1991; 13: 150-172.

11. Di Zerega GS, Campeau JD. Use of instillates to prevent intraperitoneal adhesions; crystalloid and dextran. Infertil Reprod Med Clin North Am 1994; 5: 463-478.

12. Diamond MP. Reduction of de novo postsurgical adhesions by intraoperative precoating with Seprocoat $(\mathrm{HAL}-\mathrm{C})$ solution: A prospective, randomized, blinded, placebo-controlled multicenter study. Fertil Steril 1998; 69: 1067-1074.

13. Yelimlies B, Alponat A, Cubukcu A et al. Carboxymethlcellulosecoated on visceral face of polypropylene mesh prevents adhesion without impairing wound healing in incisional hernia model in rats. Hernia 2003; 7: 130-133.

14. Avital S, Bollinger TJ,Wilkinson JD et al. Preventing intraabdominal adhesions with polylactic acid film: An animal study. Dis Col Rec 2005; 48: 153-157.

15. Hashimoto D, Hirota M, Yagi Y, Baba H. Hyaluronate carboxymethyl cellulose-based bioresorbable membrane (Seprafilm) reduces adhesion under the incision to make unplanned re-laparotomy safer. SurgToday 2012; 42: 863-867.

16. Haney AF, Doty E. Murine peritoneal injury and de novo adhesion formation caused by oxidized-regenerated cellulose (Interceed TC7) but notexpanded polytetrafluoroethylene (Gore-Tex Surgical Membrane). Fertil Steril 1992; 57: 202-208.

17. Jenkins SD, KlamerTW, Parteka JJ, Condon RE. A comparison of prosthetic materials used to repair abdominal wall defects. Surgery 1983; 94: 392-398.

18. Hooker GD, Taylor BM, Driman DK. Prevention of adhesion formation with use of sodium hyaluronate-based bioresorbable membrane in a rat model of ventral hernia repair with polypropylene mesh. A randomized, controlled study. Surgery 1999; 125: 211-216.

19. Alimoglu O, Akcakaya A, Sahin M, Ünlü Y, Özkan OV, Sanlii E, Eryilmaz R. Prevention of adhesion formation following repair of abdominal wall defects with prosthetic materials (An experimental study). HepatoGastroenterology 2003; 50: 725-728.

20. Conze J, Junge $K$, Klinge $U$ et al. Intraabdominal adhesion formation of polypropylene mesh. Surg Endosc 2005; 19: 798-803.

21. Ellis $\mathbf{H}$. Medicolegal consequences of postoperative intra-abdominal adhesions. JR Soc Med 2001; 94: 331-332

22. Miller G, Boman J, Shrier I, Gordon PH. Natural history of patients with adhesive small bowel obstruction. Br J Surg 2000; 87: 1240-1247.

23. Williams SB, Greenspon J, Young HA, Orkin BA. Small bowel obstruction: Conservative vs surgical management. Dis Col Rec 2005; 48: $1140-1146$.

Received April 21, 2014. Accepted May 5, 2014. 